\title{
Immediate Overlay Removable Partial Dentures for a Patient with Ectodermal Dysplasia: A Clinical Report
}

\author{
Ramin Negahdari ${ }^{1}$, Alireza Pournasrollah ${ }^{1}$, Seyyed Mahdi Vahid Pakde ${ }^{2^{*}}$, Sepideh Bohlouli ${ }^{3}$
}

1. Assistant Professor, Department of Prosthodontics, Faculty of Dentistry, Tabriz University of Medical Science, Tabriz, Iran

2. Post-graduate Student, Department of Prosthodontics, Faculty of Dentistry, Tabriz University of Medical Science, Tabriz, Iran

3. Assistant Professor, Department of Oral medicine, Faculty of Dentistry, Tabriz University of Medical Science, Tabriz, Iran

\begin{abstract}
Ectodermal dysplasia has several characteristic signs and symptoms, including anadontia or hypodontia, conical and hypoplastic teeth, atrophic alveolar ridges, protuberant lips, and hypotrichosis. Definitive treatment options for these patients are several including fixed, removable or implant-supported prostheses. Economic limitations or other priorities can prevent patients from choosing the most desirable treatment. This clinical report describes the prosthetic rehabilitation of a patient with ectodermal dysplasia. The treatment developed an overlay immediate removable partial denture fabricated to reconstruct acceptable occlusal vertical dimension, function and esthetics. A 2-year period of follow up showed the minor chipping of composite layer on the metal substructure.
\end{abstract}

Keywords: Ectodermal dysplasia, mouth rehabilitation

\section{Introduction}

Ectodermal dysplasia (ED) is a large and complex group of hereditary disorders defined by the abnormal development of several ectodermal structures. The most frequently reported manifestation of ED is hypohidrotic dysplasia (HED) and other type is anhydrotic dysplasia. While developing embryo, the ectoderm, gives rise to the central and peripheral nervous system, sweat glands, hair, nails, and tooth enamel $(1,2)$. Mutation in the ectodysplasin-A and ectodysplasin-A, receptor genes are responsible for $\mathrm{X}$-linked and autosomal HED (3).

The dental characteristics of this syndrome include anadontia or hypodontia, hypoplastic conical teeth and under development of the alveolar ridge $(4,5)$. Other manifestations of HED may include: hypotrichosis, hypohidrosis,

\footnotetext{
Corresponding author:

abdolhossein kazemi

Bio-medical ethic fellowship, Tabriz university of medical science, Tabriz, Iran

Email:kazemi1338@gmail.com

Receive date: 2015-10-09 | Accept date: 2015-11-18 | Publish date: 2015-12-01

DOI: 10.7575/aiac.abcmed.16.04.01.07
} 


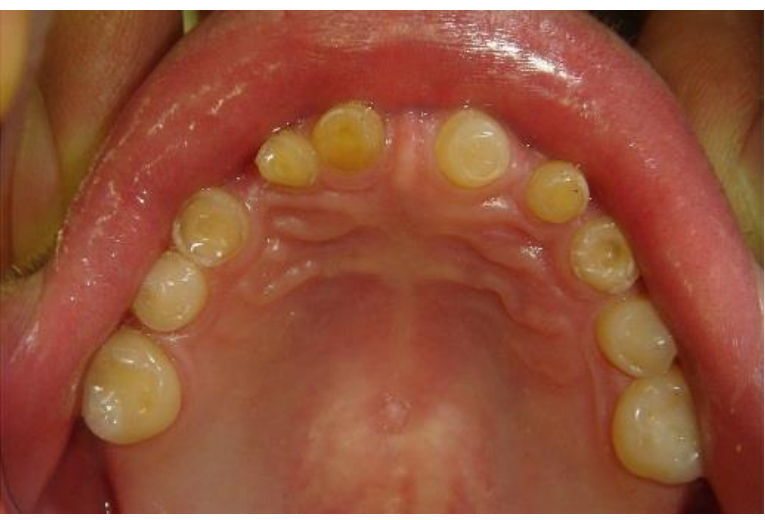

Figure 1A: Preoperative occlusal view of maxillary teeth

depressed nasal bridge, absence of sweat glands resulting in very smooth, dry skin and/or hyperkeratosis of hands and feet (6). The number of missing teeth varies, with a higher incidence in the mandible (7). In the primary dentition, maxillary second molars, canines, central incisors, and mandibular canines are the teeth most commonly present. The teeth are often conical in shape, complicating use as removable partial denture abutments (8). Oral rehabilitation of the HED patient is recommended to improve function, esthetics and psychological status (9). Prosthetic solutions for HED include RPDs, fixed partial dentures, and endo-osseous implants. RPDs is the most frequently treatment option. Overlay RPDs can preserve alveolar bone and we can achieve retention by various attachments. Some of the limitations of FPDs for HEDs are: deficient number of present teeth, large pulp size, small and conical teeth and inhibition of growth in adolescents because of the rigidity of FPDs. Implant is a desirable modality but bone quality and quantity and soft tissue contour and financial constrains can be limitations of the treatment $(4,7)$. Usually overlay denture, as an interim option can maintain occlusal integrity until the patient becomes ready for FPD or implant supported prosthesis (10).

\section{Clinical report}

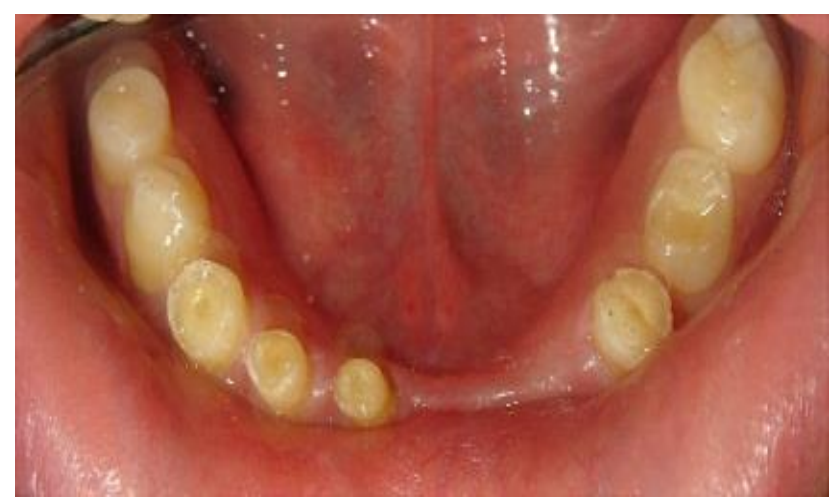

Figure 1B: Preoperative occlusal view of mandibular teeth

The patient was a 25 - year - old man. He has nail abnormality but other manifestations of HED were not present. His dental history included oligodontia in mixed dentition period. There were no permanent teeth in maxilla and he had just 10 primary teeth (incisors, canines and molars). In the mandible only the left first and second mandibular premolars and 6 primary teeth (right canine, first and second molars with left (incisors and canine) were remained as well as marked attrition of primary teeth and an underdeveloped mandibular alveolar ridge (Figure 1A, 1B).

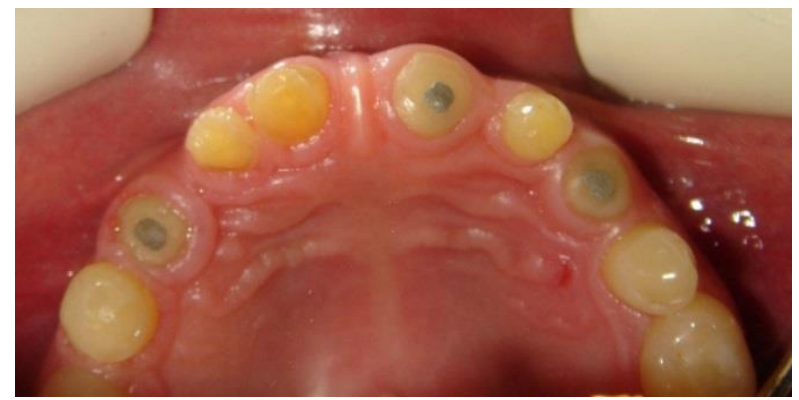

Figure 2: Root canal therapy and crown reduction of some primary teeth to have a overdenture type of RPD

The vertical height of occlusion (VDO) was decreased, as confirmed by deep folds in the commissure of the lips and over-closed appearance. Because of inadequate bone quantity and to prevent from more bone loss 
during the use of RPD we decided to reduce the crown of some primary teeth and root canal therapy was taken place for administration of an overdenture type of RPD (Left central incisor, canine and right canine) (Figure 2).

Because of emotional status of patient the extraction of the hopeless teeth (mandibular left central and lateral incisors, maxillary left central and lateral incisors and right lateral incisor) was delayed to insertion time of RPD (immediate denture). After evaluation of vertical dimension of occlusion (Free-way space: $7 \mathrm{~mm}$ ), the position of the physiological rest was determined by facial measurements and interocclusal distance. Interocclusal bite registration was done at increased vertical dimension of occlusion. Diagnostic casts were mounted in a semi adjustable articulator with use of facebow and centric relation record (Figure 3).

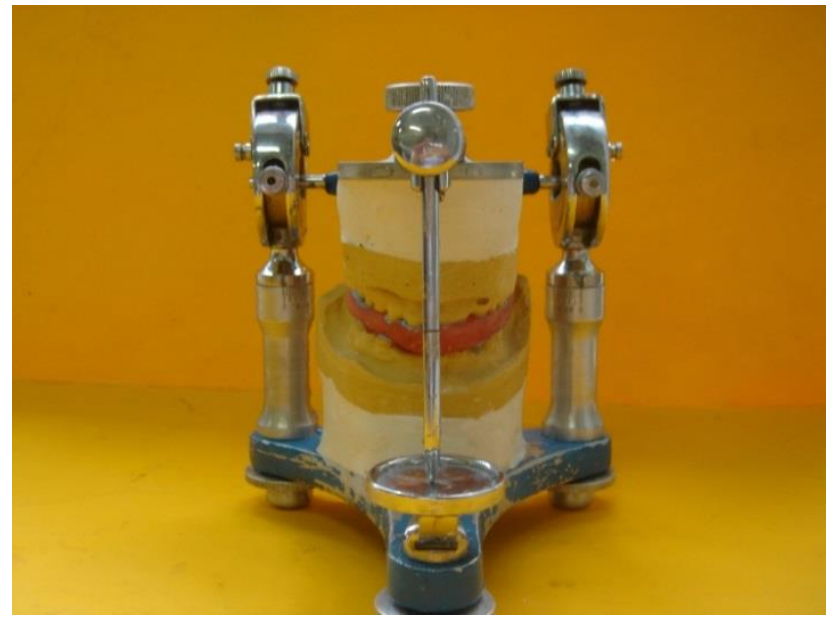

Figure 3: Diagnostic casts were mounted in semi adjustable articulator with increased vertical dimension

For determining the most appropriate path for insertion of the prosthesis, another diagnostic casts were made and surveyed. Remaining teeth have conical contours, therefore natural undercut for adequate retention frequently cannot be obtained. Consequently, the shape of the crowns was modified by applying of wax to the cast and addition of composite to the teeth to produce

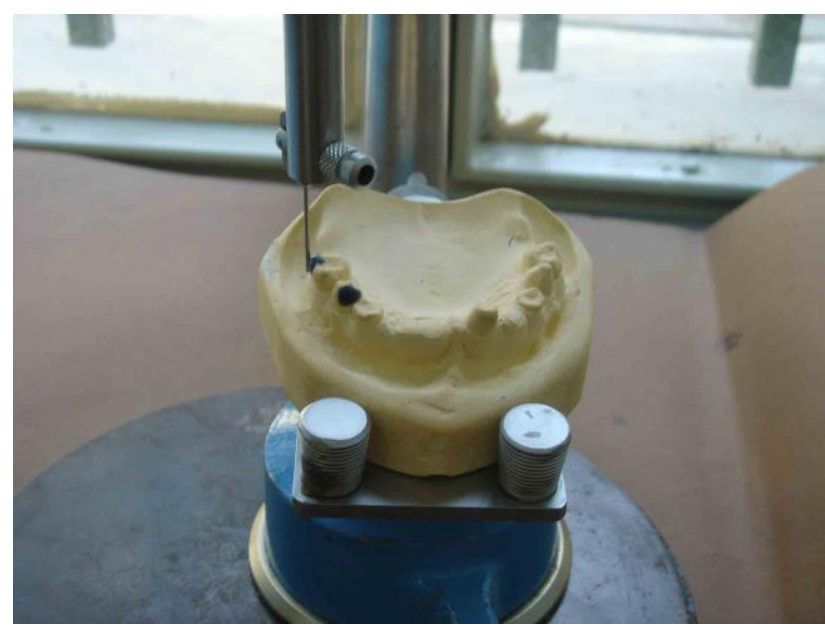

Figure 4: Surveyed diagnostic casts and crowns were modified with wax to have suitable undercuts

$0.25 \mathrm{~mm}$ undercuts (Figure 4). Because of increased vertical dimension and to have an overlay denture, onlay rest seats were prepared on the posterior abutment teeth. The teeth prescribed extraction were removed from the cast. The definitive casts were surveyed to confirm the path of insertion and the framework design was drawn. Block out of unwanted undercuts was performed before the duplication of the master cast (Figure 5A, 5B, and 5 C).

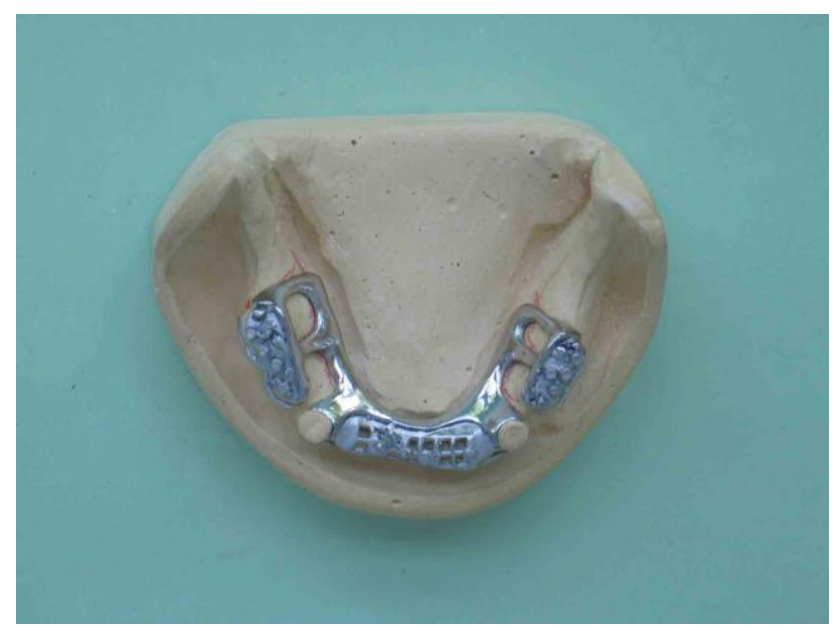

Figure 5a: Metal framework of lower overlay RPD

The maxillary refractory cast was placed in the articulator with a facebow record. An interocclusal record was used for mounting the mandibular master cast. Thereafter the CR was 


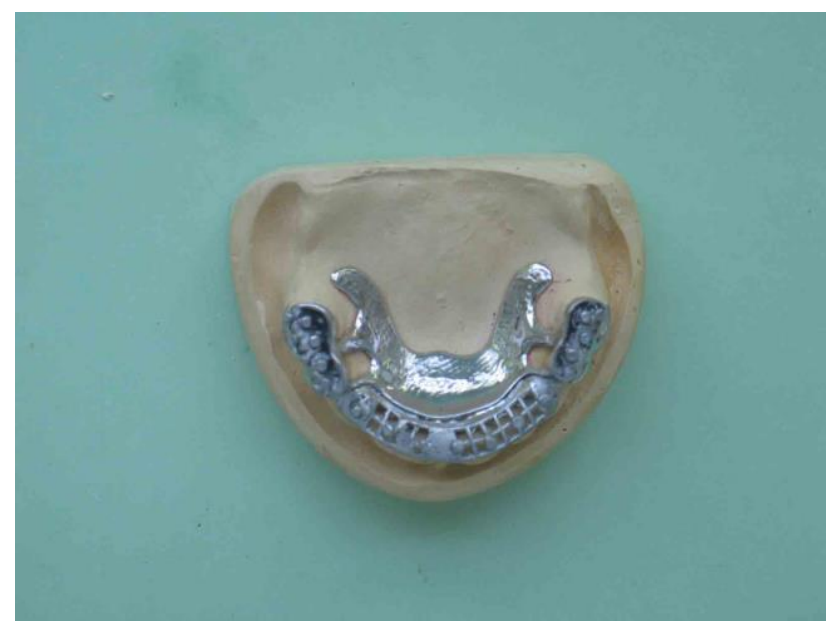

Figure 5B: Metal framework of upper overlay RPD recorded by the use of occlusion rims made on the refractory casts. During this procedure the acrylic template was used to ensure that the previous VDO and the centric relation were been reproduced. This was a key step, as it permitted the precise design of occlusion in the framework wax pattern stage. Whereas adequate interocclusal distance permits and esthetics are paramount, the occlusal surface may be formed with composite with a metal substructure. The effect of occlusion in metal occlusal surfaces maybe magnified and can be damaging not only to opposing teeth but also to periodontal ligament and the supporting bone. The pattern was invested, cast in a chrome - cobalt alloy, and finished.

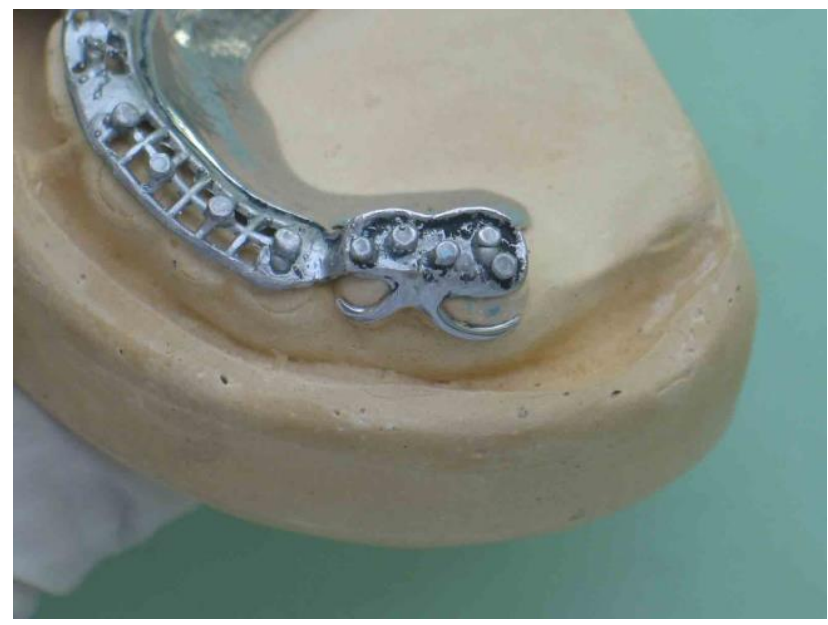

Figure 5C: Onlay rests and retentive elements for composite
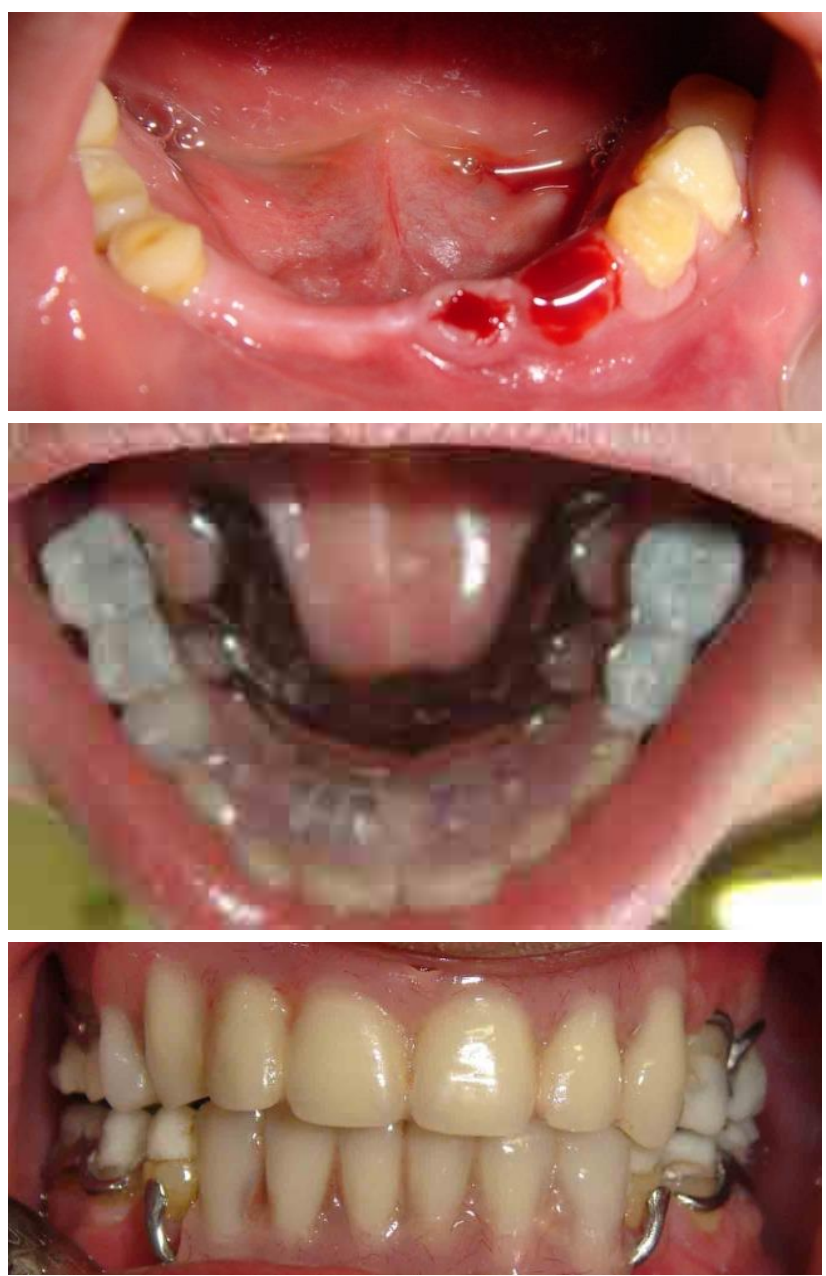

Figure 6: Extraction of the teeth must be extracted and delivery of the immediate overlay RPD

When the framework was ready, because of the immediate nature of RPD, it is not possible to evaluate framework intraorally. The frameworks seated on master cast, were mounted with the same interocclusal record in a semi adjustable articulator then the artificial teeth were arranged for an esthetic and functional purposes according the anatomic landmarks and midline position which is marked on interocclusal record. After processing, the casts were remounted and the occlusion was adjusted to eliminate processing errors. Visible light - polymerized composite was used for the fabrication of occlusal surfaces on the removable partial metal framework. After extracting the hopeless teeth, the 
maxillary and mandibular RPDs were inserted with minor occlusal adjustments (Figure 6).

The patient was educated how to properly insert and remove the prosthesis and give instructions on adequate oral hygiene and use of fluoride mouth wash. Patient be wanted don't remove his prosthesis during $24-48$ hours due to the possibility of swelling of inflammatory tissues. Regular recalls were scheduled for 2 years to make necessary adjustments and monitor the patient's cooperation and oral hygiene. During the follow up period the minor chipping of composite layer on the metal substructure was shown (Figure 7).

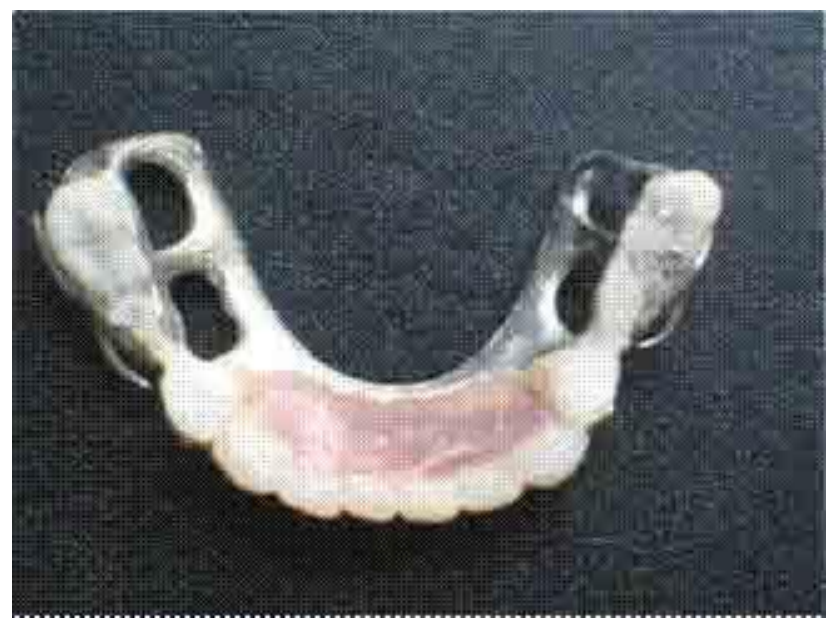

Figure 8: Chipping of composite layer on the metal substructure at the follow up period

\section{Discussion}

Hypodontia of primary and permanent dentition is one of the most frequent symptoms in patients with hereditary ectodermal dysplasia (HED). These features of poor esthetic affect the social and the psychological well-being of the patient. A prosthetic rehabilitation of a HED patient aims to improve esthetic and oral functions, as well as to improve psychological and social comfort to the child, who will remain under dentistry treatment until it is completely grown-up and orally developed $(5,11,12)$.
Dental rehabilitation of a patient with HED could be performed by three modalities: fixed prosthesis (tooth supported FDPs), removable prosthesis, and implant supported prosthesis. Since many individuals have a minimal number of teeth, tooth supported FDPs are rarely used exclusively in the treatment of ED. Removable prosthesis is treatment of choice for the dental management of ED's. Because anodontia or hypodontia is typical in individuals with this condition, complete dentures, partial dentures, or overdentures are often part of the treatment provided. Although complete dentures can provide an acceptable results, underdevelopment of the edentulous alveolar ridges in individuals with ED can compromise denture retention and stability. Remained teeth can be used for support overdentures with the advantages of preservation of alveolar bone. Implant supported prosthesis is alternative treatment option which provide much more advantages but has some difficulties in growing younger patients and need better financial support (4).

The overlay removable denture is a reversible and cost-effective treatment option for patients and can be modified periodically for pleasing the patient (13-15). The advantages of overlay removable dentures include preservation of the residual ridge, support and stabilization of their base, improving the soft tissue profile and the aesthetic aspect, and giving patients a sense of security in knowing that teeth do support their prostheses (10).

In this specific case the immediate overlay removable partial denture was employed for oral rehabilitation because of mentioned advantages and patient's economic problems for implant supported prosthesis. Several case reports had employed this approach for oral rehabilitation of HED, worn teeth, amelogenesis imperfecta, and reestablishment of occlusion (7, 10, 13-20). In all previous 
reports the overlay denture was fabricated after extraction of hopeless teeth but in our case, because of the decreased self-confidence and self-steam of the patient and the negative effect of teeth extraction on emotional status of him, immediate overlay denture was fabricated which needs extra technically sensitive procedures for fabrication. Immediate denture was fabricated after cast surgery and relined in periodic recalls for better adaptation. Since the patient growth was terminated, we did not have adjusting problems regarding jaw growth. Prosthetic rehabilitation improves esthetics and function and plan of occlusion would be more favorable (Figure 8). The patient's social confidence also improved significantly as a result of dental treatment.

This kind of treatment surely has some disadvantages such as complaints related to less esthetic when the dentures are removed, as well oral comfort. Another complication associated with overlay treatment is the high possibility to caries and periodontal disease adjacent to the abutments even if prevented measures are applied. Nonetheless, these problems can be some manifestations of poor oral hygiene. Other risks of removal partial dentures are mechanical complications such as fractures of major or minor connector, occlusal
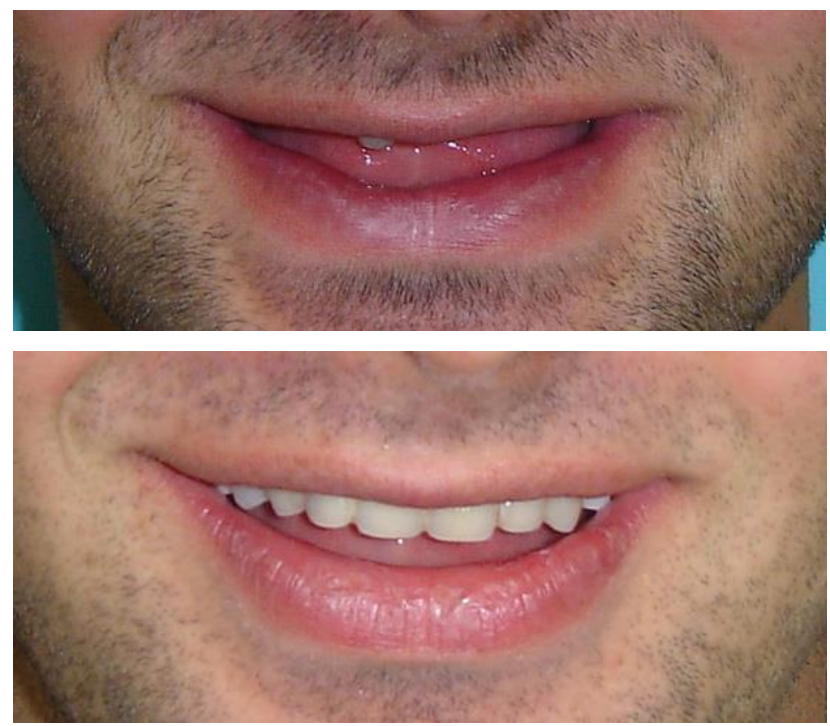

Figure 8: Improved esthetics of the patient after delivery of the immediate overlay RPD

rests deformation or fracture. Yet, the advantages associated with the kind of treatment manipulate in this study conquest on the problems of it. However, supplementary studies should be performed to evidence the overlay treatments.

\section{Conclusions}

This clinical report demonstrated that immediate overlay RPDs can be a reversible, bone preserver and inexpensive treatment modality for ectodermal dysplasia patients with a reduced number of teeth and financial constraints.

\section{References}

1. Abadi B, Herren C. Clinical treatment of ectodermal dysplasia: a case report. Quintessence international. 2001;32(9):743-5.

2. Lamartine J. Towards a new classification of ectodermal dysplasias. Clinical and experimental dermatology. 2003;28(4):351-5.

3. Jaskoll T, Zhou YM, Trump G, Melnick M. Ectodysplasin receptor-mediated signaling is essential for embryonic submandibular salivary gland development. The anatomical record Part A, Discoveries in molecular, cellular, and evolutionary biology. 2003;271(2):322-31.

4. Pigno MA, Blackman RB, Cronin RJ, Jr., Cavazos E. Prosthodontic management of ectodermal dysplasia: a review of the literature. The Journal of prosthetic dentistry. 1996;76(5):541-5.

5. Bergendal B. Orodental manifestations in ectodermal dysplasia-A review. American journal of medical genetics Part A. 2014. 
6. Priolo M, Silengo M, Lerone M, Ravazzolo R. Ectodermal dysplasias: not only 'skin' deep. Clinical genetics. 2000;58(6):415-30.

7. Ghodsi S, Rasaeipour S, Vojdani M. Oral rehabilitation of a patient with amelogenesis imperfecta using removable overlay denture: a clinical report. The journal of contemporary dental practice. 2012;13(2):227-31.

8. Abadi BJ, Kimmel NA, Falace DA. Modified overdentures for the management of oligodontia and developmental defects. ASDC journal of dentistry for children. 1982;49(2):123-6.

9. Pope AW, Ward J. Factors associated with peer social competence in preadolescents with craniofacial anomalies. Journal of pediatric psychology. 1997;22(4):455-69.

10. Pavarina AC, Machado AL, Vergani CE, Giampaolo ET. Overlay removable partial dentures for a patient with ectodermal dysplasia: a clinical report. The Journal of prosthetic dentistry. 2001;86(6):574-7.

11. Bergendal B. Prosthetic habilitation of a young patient with hypohidrotic ectodermal dysplasia and oligodontia: a case report of 20 years of treatment. The International journal of prosthodontics. 2001;14(5):471-9.

12. Paschos E, Huth KC, Hickel R. Clinical management of hypohidrotic ectodermal dysplasia with anodontia: case report. The Journal of clinical pediatric dentistry. 2002;27(1):5-8.

13. Ganddini MR, Al-Mardini M, Graser GN, Almog D. Maxillary and mandibular overlay removable partial dentures for the restoration of worn teeth. The Journal of prosthetic dentistry. 2004;91(3):210-4.

14. Del Castillo R, Lamar F, Jr., Ercoli C. Maxillary and mandibular overlay removable partial dentures for the treatment of posterior open-occlusal relationship: a clinical report. The Journal of prosthetic dentistry. 2002;87(6):587-92.

15. Fonseca J, Nicolau P, Daher T. Maxillary overlay removable partial dentures for the restoration of worn teeth. Compendium of continuing education in dentistry. 2011;32(3):12, 4-20; quiz 1, 32.

16. Beyth N, Tamari I, Buller Sharon A. Overlay removable denture for treatment of worn teeth. Special care in dentistry : official publication of the American Association of Hospital Dentists, the Academy of Dentistry for the Handicapped, and the American Society for Geriatric Dentistry. 2014.

17. Bataglion C, Hotta TH, Matsumoto W, Ruellas CV. Reestablishment of occlusion through overlay removable partial dentures: a case report. Brazilian dental journal. 2012;23(2):172-4.

18. Patel MB, Bencharit S. A treatment protocol for restoring occlusal vertical dimension using an overlay removable partial denture as an alternative to extensive fixed restorations: a clinical report. The open dentistry journal. 2009;3:213-8.

19. Almog DM, Ganddini MR. Maxillary and mandibular overlay removable partial dentures for restoration of worn teeth. A three-year follow-up. The New York state dental journal. 2006;72(3):32-5.

20. Sato S, Hotta TH, Pedrazzi V. Removable occlusal overlay splint in the management of tooth wear: a clinical report. The Journal of prosthetic dentistry. 2000;83(4):392-5. 